\title{
Structural Description of the Macroscopic Piezo- and Ferroelectric Properties of Lead Zirconate Titanate
}

\author{
M. Hinterstein, ${ }^{1}$ J. Rouquette,, $2 *$ J. Haines,${ }^{2}$ Ph. Papet, ${ }^{2}$ M. Knapp, ${ }^{3}$ J. Glaum,,${ }^{4,5}$ and H. Fuess ${ }^{4}$ \\ ${ }^{1}$ Institut für Werkstoffwissenschaften, Technische Universität Dresden, Helmholtzstrasse 7, D-01069 Dresden, Germany \\ ${ }^{2}$ Institut Charles Gerhardt UMR CNRS 5253 Equipe $C_{2} M$, Université Montpellier II, \\ Place Eugene Bataillon, cc1504, 34095 Montpellier cedex 5, France \\ ${ }^{3}$ CELLS-ALBA, Carretera BP 1413, Km. 3.3, E-08290 Cerdanyola (Barcelona), Spain \\ ${ }^{4}$ Institute for Materials Science, Technische Universität Darmstadt, Petersenstrasse 23, D-64287 Darmstadt, Germany \\ ${ }^{5}$ School of Materials Science and Engineering, University of New South Wales, Sydney, NSW, Australia
}

(Received 1 April 2011; published 9 August 2011)

\begin{abstract}
An in situ structural description of the origin of the ferroelectric properties as a function of the applied electric field $E$ was obtained by synchrotron x-ray diffraction. A setup was used to average the effects of the preferred orientation induced by the strong piezoelectric strain and solve in situ the crystal structure as a function of the applied electric field. Hence, we were able to describe the microscopic origin of the macroscopic ferro- and piezoelectric properties of the most widely used ferroelectric material, lead zirconate titanate.
\end{abstract}

DOI: 10.1103/PhysRevLett.107.077602

PZT materials with compositions at the morphotropic phase boundary (MPB) between the tetragonal $(P 4 \mathrm{~mm})$ and rhombohedral $(R 3 m)$ phase fields exhibit very high dielectric and piezoelectric properties which are intensively used for technological applications (sensors and actuators, microelectromechanical systems, and high frequency devices) [1]. The origin of these effects is either described by (1) the coexistence of rhombohedral and tetragonal phases that can be linked with the martensitic phase transition theory [2] or by (2) the existence of a monoclinic $\mathrm{Cm}$ phase [3].

In the martensitic transition theory, high piezoelectric response is explained by reorientation of complex nanodomain structures, whereas the presence of a monoclinic phase in the PZT phase diagram gives rise to the polarization rotation mechanism [4]. Although these mechanisms offer an interesting zero-field description of the microscopic properties, only the entire in situ structural picture of the ferroelectric properties as a function of the applied electric field can provide the fundamental information necessary to understand these materials in their technological application.

However, application of an electric field results in the creation of a large strain linked to the piezoelectric effect [5] $\left(\partial d_{k i j}=\frac{\partial \eta_{i j}}{\partial E_{k}}\right.$, where $d_{k i j}$ is the piezoelectric coefficient, $\eta_{i j}$ is the strain, and $E_{k}$ is the applied electric field); preferred orientation has to be taken into account in the Rietveld refinement, which makes structure refinement challenging [6]. This is why in the literature only the individual electric-field-induced $h k l$ lattice strains $\eta_{h k l}$ give objective information [7-10], i.e., $\eta_{h k l}=$ $\left(d_{h k l}^{E}-d_{h k l}^{0}\right) / d_{h k l}^{0}$, where $d_{h k l}^{E}$ and $d_{h k l}^{0}$ are the $(h k l)$ lattice spacing with and without field, respectively.
PACS numbers: 77.84.Cg, 61.05.C-, 77.65.-j, 77.80.Fm

Note, however, that as the sample becomes poled under field it becomes strained and additional electric field cycling will be obscured by the remanent electric-fieldinduced strain for most of the structural reflections, i.e., particularly the pseudocubic $\{002\}_{c}$ reflections which are known to be quite sensitive to texturing [11]. The subscript " $c$ " denotes the pseudocubic perovskite unit cell. In this study, an appropriate setup was used to minimize the effects of the preferred orientation and solve in situ the crystal structure as a function of the applied electric field. Hence, we were able to describe microscopically the macroscopic physical properties of commercial ferroelectric PZT.

In order to investigate the cyclic poling process of PZT, we performed $\mathrm{x}$-ray measurements at the high resolution powder diffraction station of the materials science beam line [12] at the Swiss Light Source (SLS, Villigen, Switzerland) using high-energy photons $\lambda=0.443187 \AA$ to study thin metallized PZT pellets (thickness $<100 \mu \mathrm{m}$ ) in transmission geometry. Commercially available PIC 151 (PI Ceramics, Lederhose, Germany), a soft-doped PZT with a composition near the MPB, was used as it presents optimal piezo- and ferroelectric properties $\left(d_{33}=\right.$ $500 \mathrm{pC} / \mathrm{N}$ ), long operation periods, and offers a high degree of reproducibility. For the experiment, a modified high voltage sample environment from beam line B2 at HASYLAB at DESY was installed [6]. The utilized MYTHEN II detector [13] provided data with an extremely high signal-to-noise ratio and high resolution together with a low acquisition time of $10 \mathrm{~s}$ per diffraction pattern. The time to measure a complete ferroelectric hysteresis cycle was about $20 \mathrm{~min}$.

Because of the diffraction conditions an experiment at an angle $\psi=0^{\circ}$ is dominated by $\eta_{y}$, whereas $\psi=90^{\circ}$ 
favors $\eta_{x}$. Therefore, the sample was mounted at $\psi=45^{\circ}$ to minimize texture effects, because then the contribution of $\eta_{x}$ is similar to the one from $\eta_{y}$ [Fig. 1(a)]. This simple setup permits us to keep the intensity ratio of the tetragonal $200_{t} / 002_{t}$ reflection pair in the appropriate random distribution even for a poled sample, which is a good indication of the absence of preferred orientation effects in the diffractogram [Fig. 1(b)]. Figure 2 shows the influence of the applied electric field on this tetragonal reflection pair. It is consistent with a decrease in the tetragonal intensity while the rhombohedral-like intensity in between the tetragonal reflections is increasing with electric field.

Rietveld refinements using the program FULLPROF [14] were successfully performed to describe the structural evolution as a function of the applied electric field over the entire hysteresis cycle, i.e., $-2.2 \leq E \leq 2.2 \mathrm{kV} / \mathrm{mm}$ range, without any preferred orientation correction. Note that due to this geometry, the applied cyclic electric field $(-2.2 \leq E \leq 2.2 \mathrm{kV} / \mathrm{mm})$ resulted in a perfect reversibility of the x-ray diffractograms, which clearly demonstrates the absence of a macroscopic remanent strain in the patterns (Fig. 2).

Monoclinic-tetragonal phase coexistence and microstrains $[15,16]$ were used for the structural model. As a

(a)
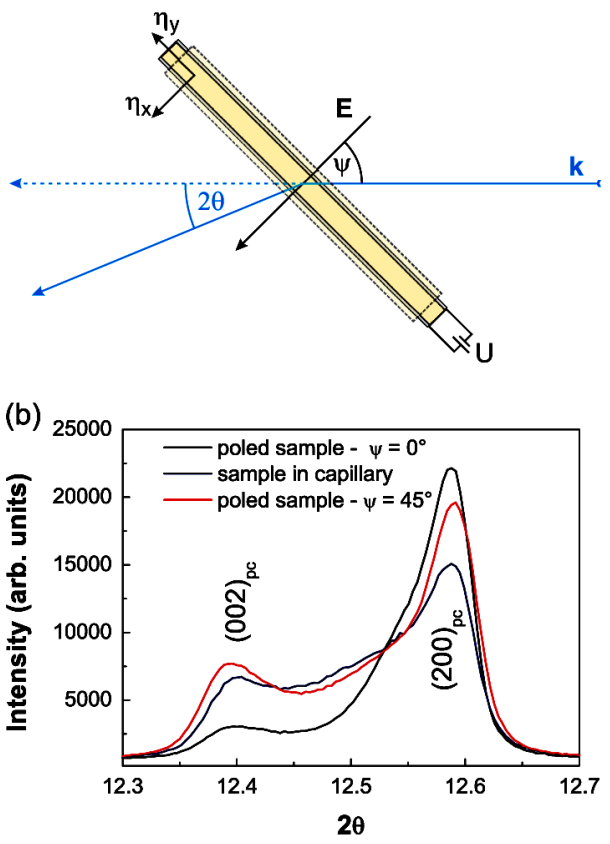

FIG. 1 (color online). (a) $45^{\circ}$-scattering geometry used in the experiment. Note that the effects texturing $\left(\eta_{x}=\eta_{y}\right)$ induced by remanent strain are minimized in such a configuration. For better visualization the field induced strain is exaggerated. (b) The $200_{t} / 002_{t}$ tetragonal reflection pair intensity for a PZT sample in a capillary (blue line), for poled sample with $0^{\circ}$-scattering geometry (black line), and for a poled sample with $45^{\circ}$-scattering geometry (red line). The preferred orientation is characterized by a strong deviation of the intensity ratio $\left(\psi=0^{\circ}\right)$. rhombohedral model could not correctly reproduce the low-field data, this structural model was abandoned. Dependent on the structural parameters, the monoclinic phase can be interpreted as tetragonal, monoclinic, or rhombohedral [16]. To minimize the number of variables and stabilize the refinements, tetragonal microstrains were kept fixed for the applied cyclic electric field. Figure 3 shows the refined x-ray diffractogram of poled PZT through the continuous sinusoidal acquisition obtained at 0 and $1 \mathrm{kV} / \mathrm{mm}$, respectively. The associated refined structural parameters can be found in Table 1 of the Supplemental Material [17]. Because of the $45^{\circ}$-scattering geometry no preferred orientation is detected as mentioned above. All the typical characteristics, i.e., the changes in the $\{002\}_{c}$ reflections as well as high- $2 \theta$ data, are perfectly reproduced in the range $-2.2 \leq E \leq 2.2 \mathrm{kV} / \mathrm{mm}$.

These Rietveld refinements have enabled us to give a microscopic description of the properties of the ferroelectric hysteresis loop. Figure 4 shows some examples for the structural behavior of PZT as a function of the applied electric field. Figure 4(a) exhibits the tetragonalmonoclinic relative abundance with electric field. Note that the monoclinic phase exhibits the highest abundance in the whole electric field range; thus, the discussion of the structural behavior will mainly be based on this phase. The monoclinic content first increases by about $15 \%$ at $0.6 \mathrm{kV} / \mathrm{mm}$ [(1) in the hysteresis cycle (HC)]. Above $1 \mathrm{kV} / \mathrm{mm}$, the content saturates to about $75 \%$ at $2.2 \mathrm{kV} / \mathrm{mm}$. When the field decreases, (2) in $\mathrm{HC}$, the monoclinic abundance continuously decreases even when the sign of the electric field is reversed, (3) in $\mathrm{HC}$, and will only increase again above $-0.7 \mathrm{kV} / \mathrm{mm}$ with a $15 \%$ jump centered at $-0.9 \mathrm{kV} / \mathrm{mm}$. Again the monoclinic content saturates at about $75 \%$ above $-1.2 \mathrm{kV} / \mathrm{mm}$. When the
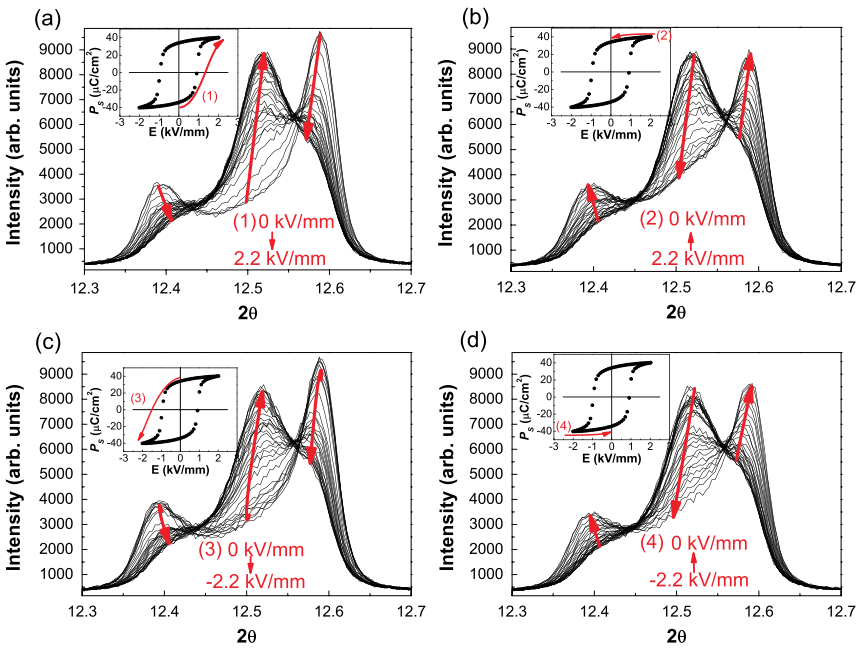

FIG. 2 (color online). Influence of the applied electric field on the $002_{t}$ reflection, (a) from 0 to $2.2 \mathrm{kV} / \mathrm{cm}$, (b) from 2.2 to $0 \mathrm{kV} / \mathrm{cm}$, (c) from 0 to $-2.2 \mathrm{kV} / \mathrm{cm}$, and (d) from -2.2 to $0 \mathrm{kV} / \mathrm{cm}$. The insets show the position in the corresponding hysteresis cycle (HC) for the same range of electric field (1)-(4). 
(a)

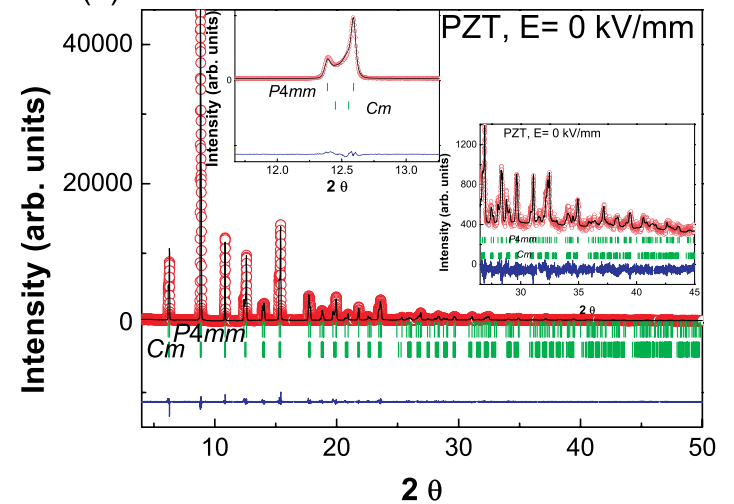

(b)

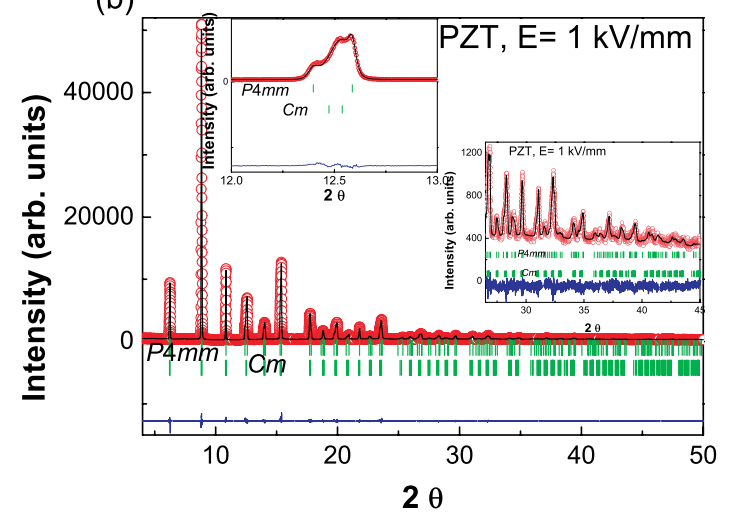

FIG. 3 (color online). (a) Rietveld refinements of PZT at $0 \mathrm{kV} / \mathrm{mm}$ and (b) $1 \mathrm{kV} / \mathrm{mm}$. The difference plot has same scale. The vertical tick marks indicate the calculated positions of the $P 4 \mathrm{~mm}$ phase (top) and $\mathrm{Cm}$ phase reflections (bottom). The insets show the corresponding $\mathrm{HC}$ for the same range of electric field (1)-(4).

applied electric field decreases, (4) in $\mathrm{HC}$, the content decreases again to an abundance of $63 \%$ at $0 \mathrm{kV} / \mathrm{mm}$.

However, the refinements are of high quality as the relative abundance is consistent with a butterfly shape, which is a characteristic macroscopic signature of these materials [1] and can be found, for instance, in field-strain and field-dielectric constant diagrams. Additionally, it is obvious that the $15 \%$ jump, which occurs at 0.6 and $-0.9 \mathrm{kV} / \mathrm{mm}$, respectively, is consistent with the $0.8 \mathrm{kV} / \mathrm{mm}$ coercive field of the material, i.e., the electric field value required to flip the spontaneous polarization. Finally, Fig. 4(a) gives interesting information on the origin of the MPB properties, i.e., the martensitic phase transition theory [2] versus the existence of a monoclinic $\mathrm{Cm}$ phase [3]. If the martensitic model was correct, the nanotwins should detwin under field and the phase fraction of tetragonal phase should increase with respect to the monoclinic or twinned phase, which is opposite of what is observed. Therefore, our results strongly support the existence of a monoclinic symmetry for the MPB in PZT as a function of the applied electric field. An extensive transmission electron microscopy study by convergent
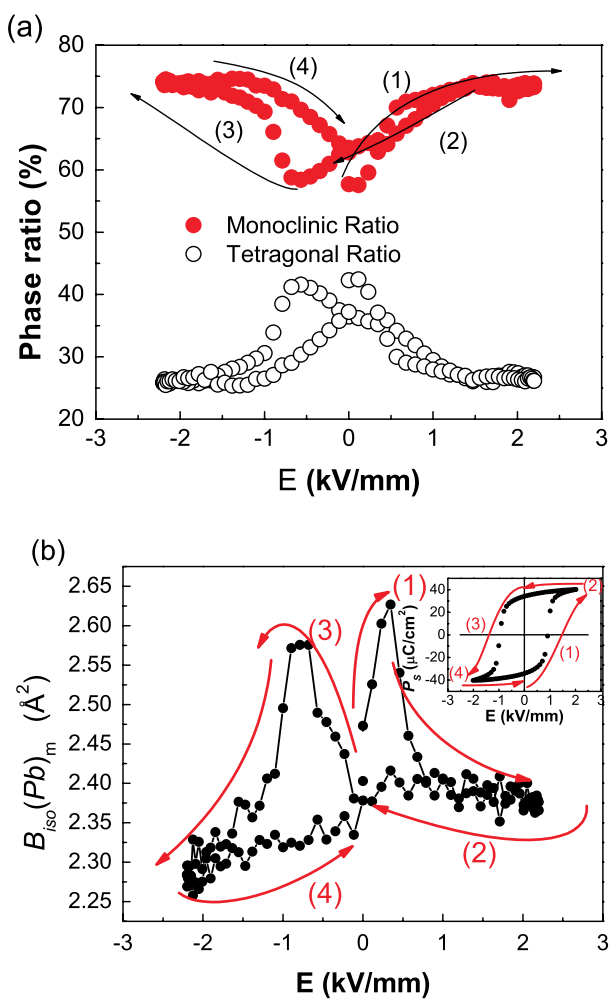

FIG. 4 (color online). (a) Monoclinic-tetragonal phase fraction $(\%)$ and (b) isotropic mean square displacement of the lead atom in the monoclinic phase $B_{\text {iso }}(\mathrm{Pb})_{m}$. The inset shows the corresponding $\mathrm{HC}$ for the same range of electric field (1)-4).

beam electron diffraction [18] across the MPB provides further evidence of the existence of monoclinic symmetry within microdomains.

The high quality and reproducibility of the Rietveld refinements is further demonstrated by the electric field dependence of the mean square displacement of the lead atom $\left[B_{\text {iso }}(\mathrm{Pb})_{m}\right]$ in the monoclinic phase [Fig. 4(b)]. This structural parameter is associated with the disorder of the considered atom [19]; it is, therefore, very sensitive to any structural change. Hence, its electric field dependence and dispersion can be viewed as a good measure of the quality of the Rietveld refinements.

Based on the results described above, the monoclinic phase was found to be the most representative, particularly in the high electric field range. Of particular importance is the high accuracy of the refined $B_{\text {iso }}(\mathrm{Pb})_{m}$. This is due to the large $d$-range spacing $(0.5-6.3 \AA)$ obtained using $\lambda=0.443187 \AA$ in our experiment. In addition and due to the hard $\mathrm{x}$ rays used, the scattered intensity is dominated by the lead atoms. $B_{\text {iso }}(\mathrm{Pb})_{m}$ increases with electric field, (1) in HC, and reaches a maximum of about $2.63 \AA^{2}$ at $0.5 \mathrm{kV} / \mathrm{mm}$.

Above this field, $B_{\text {iso }}(\mathrm{Pb})_{m}$ returns back to a value of about $2.4 \AA^{2}$ and stays there up to the highest electric field. When the field decreases, (2) in $\mathrm{HC}, B_{\text {iso }}(\mathrm{Pb})_{m}$ stays constant until the field is reversed. Then, (3) in HC, the mean 
squared displacement exhibits again a maximum at about $-0.9 \mathrm{kV} / \mathrm{mm}$ and decreases to a value of $2.25 \AA^{2}$ at the maximum field in the opposite direction. When the negative field goes back to zero, (4) in $\mathrm{HC}, B_{\text {iso }}(\mathrm{Pb})_{m}$ increases smoothly to $2.4 \AA^{2}$ without any further anomaly.

It is interesting to note that the anomalies in the mean square displacement of lead are always linked to a change in sign of the spontaneous polarization [(1) and (3) in $\mathrm{HC}$ ]. It means that the polarization flipping can unambiguously be detected by our structural study. One has to keep in mind that lead atoms are located in noncentrosymmetric positions; i.e., they have polar displacements in the PZT structure. It is, therefore, reasonable to associate the polarization flipping carried out by these polar atoms with a maximum in the disorder parameter of lead; i.e., $B_{\text {iso }}(\mathrm{Pb})_{m}$ characterizes the maximum of the entropy. Note that this effect can also be observed for lead atoms in the tetragonal phase, but is less pronounced due to the small phase fraction. In addition, this anomaly could also clearly be observed in the other structural parameters as, for instance, the electric field dependence of the atomic positions and unit cell parameters. The refined structural parameters were finally used to calculate the piezoelectric properties of the PZT sample. The calculated value of the piezoelectric coefficient $d_{33}$ of PZT based on the structural parameters obtained in the Rietveld refinements and macroscopic measurements of $d_{33}$ are in remarkable qualitative agreement; see the Supplemental Material [17].

In conclusion, in situ x-ray diffraction experiments on high performance PZT materials as a function of an applied electric field were performed with a $45^{\circ}$-scattering geometry. Thus we minimized the effects of preferred orientation and carried out reliable Rietveld refinements. The application of an electric field revealed an increase of the monoclinic phase fraction. During the bipolar cycling the structure shows a fully reversible switching between zero field and maximum field resulting in a relative abundance plot with a butterfly shape similar to the characteristic macroscopic strain loop [Fig. 4(a)]. Note that this setup could be used in other studies, involving homogeneous strains as a function of other intrinsic physical parameters, i.e., stress, magnetic field, etc. The structural information obtained provides an accurate microscopic description of the macroscopic piezoand ferroelectric properties of PZT materials. Such information is a major goal of materials science studies and gives the appropriate structural picture of a functional material under in situ conditions as they occur in technological applications. These results will be useful for the design and optimization of higher performance materials.

This work was performed at the Swiss Light Source, Paul Scherrer Institute, Villigen, Switzerland. The authors would like to thank F. Gozzo and A. Cervellino for the beam line support and A. Bergamaschi for the competent help with the MYTHEN detector system. The research leading to these results has received funding from the
BMBF (Bundesministerium für Bildung und Forschung) project Grant No. 05K10ODA and from the European Community's Seventh Framework Programme FP7/20072013 under Grant Agreement No. 226716. The authors appreciate the financial support of the German Research Foundation (DFG) through the Sonderforschungsbereich 595 "Electric fatigue in functional materials" and the German Crystallographic Society (DGK).

*jerome.rouquette@univ-montp2.fr

[1] B. Jaffe, W. R. Cook, and H. Jaffe, Piezoelectric Ceramics (Academic, London, 1971).

[2] Y. M. Jin, Y. U. Wang, A. G. Khachaturyan, J. F. Li, and D. Viehland, J. Appl. Phys. 94, 3629 (2003).

[3] B. Noheda, D. E. Cox, G. Shirane, J. A. Gonzalo, L. E. Cross, and S.-E. Park, Appl. Phys. Lett. 74, 2059 (1999).

[4] R. Guo, L. E. Cross, S.-E. Park, B. Noheda, D. E. Cox, and G. Shirane, Phys. Rev. Lett. 84, 5423 (2000).

[5] M. E. Lines and A. M. Glass, Principles and Applications of Ferroelectrics and Related Materials (Clarendon, Oxford, 1977).

[6] K. A. Schonau, M. Knapp, H. Kungl, M. J. Hoffmann, and H. Fuess, Phys. Rev. B 76, 144112 (2007).

[7] A. Pramanick, J. Daniels, and J. Jones, J. Am. Ceram. Soc. 92, 2300 (2009).

[8] D. Hall, A. Steuwer, B. Cherdhirunkorn, T. Mori, and P. Whiters, J. Appl. Phys. 96, 4245 (2004).

[9] J. Jones, A. Pramanick, J. Nino, S. Motahari, M. Daymond, and E. Oliver, Appl. Phys. Lett. 90, 172909 (2007).

[10] D. A. Hall, A. Steuwer, B. Cherdhirunkorn, T. Mori, and P. J. Withers, J. Appl. Phys. 96, 4245 (2004).

[11] A. Pramanick, A. Prewitt, M. Cottrell, W. Lee, A. Studer, K. An, C. Hubbard, and J. Jones, Appl. Phys. A 99, 557 (2010).

[12] B. Patterson et al., Nucl. Instrum. Methods Phys. Res., Sect. A 540, 42 (2005).

[13] A. Bergamaschi, A. Cervellino, R. Dinapoli, F. Gozzo, B. Henrich, I. Johnson, P. Kraft, A. Mozzanica, B. Schmitt, and X. Shi, J. Synchrotron Radiat. 17, 653 (2010).

[14] J. Rodriguez-Carvajal, Physica (Amsterdam) 192B, 55 (1993).

[15] J. Frantti, S. Eriksson, S. Hull, V. Lantto, H. Rudlöf, and K. Kakihana, J. Phys. Condens. Matter 15, 6031 (2003).

[16] M. Hinterstein, K. A. Schoenau, J. Kling, H. Fuess, M. Knapp, H. Kungl, and M. J. Hoffmann, J. Appl. Phys. 108, 024110 (2010).

[17] See Supplemental Material at http://link.aps.org/ supplemental/10.1103/PhysRevLett.107.077602 for a description of the electric field dependence of the structural data obtained by Rietveld refinements. These parameters are used to calculate the piezoelectric properties of the PZT sample.

[18] R. Schierholz and H. Fuess (to be published); R. Schierholz, H. Fuess, K. Tsuda, Y. Ogata, M. Terauchi, and R. Theissmann, Phys. Rev. B 78, 024118 (2008).

[19] A. Al-Zein, G. Fraysse, J. Rouquette, P. Papet, J. Haines, B. Hehlen, C. Levelut, G. Aquilanti, and Y. Joly, Phys. Rev. B 81, 174110 (2010). 